\title{
Subregional DXA-derived vertebral bone mineral measures are stronger predictors of failure load in specimens with lower areal bone mineral density, compared to those with higher areal bone mineral density
}

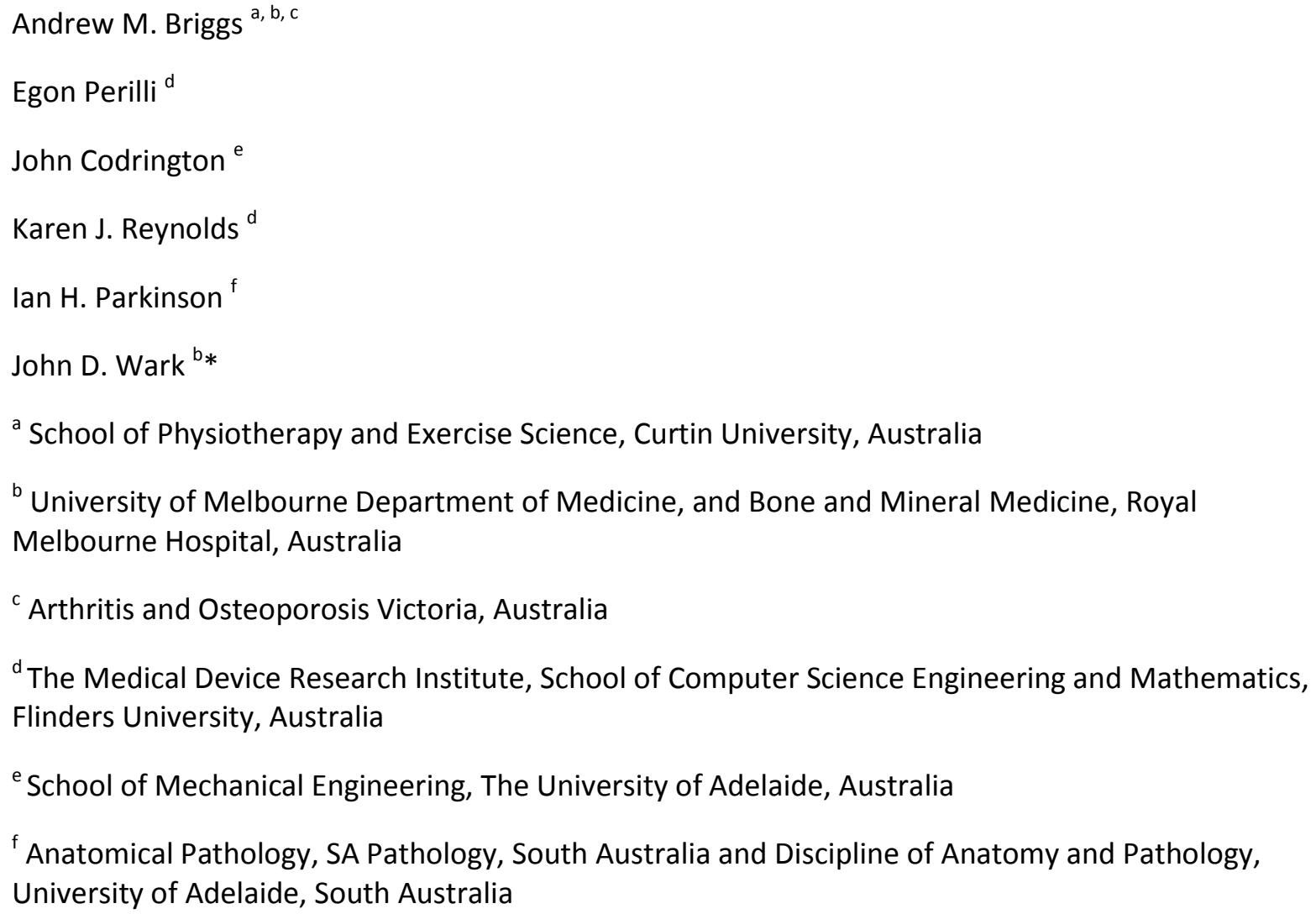




\section{Abstract}

Measurement of areal bone mineral density $(\mathrm{aBMD})$ in intra-vertebral subregions may increase the diagnostic sensitivity of dual energy X-ray absorptiometry (DXA)-derived parameters for vertebral fragility. This study investigated whether DXA-derived bone parameters in vertebral subregions were better predictors of vertebral bone strength in specimens with low aBMD, compared to those with higher aBMD.

Twenty-five lumbar vertebrae (15 embalmed, 10 fresh-frozen) were scanned with postero-anterior (PA) and lateral-projection DXA and then mechanically tested in compression to ultimate failure. Whole-vertebral aBMD and bone mineral content $(\mathrm{BMC})$ were measured from the PA- and lateralprojection scans and within 6 intra-vertebral subregions. Multivariate regression was used to predict ultimate failure load by BMC, adjusted for vertebral size and specimen fixation status across the whole specimen set, and when sub-grouped into specimens with low aBMD and high aBMD.

Adjusted BMC explained a substantial proportion of variance in ultimate vertebral load when measured over the whole vertebral area in lateral projection (adjusted $R^{2}: 0.84$ ) and across the six subregions (ROIs 2-7) (adjusted $\mathrm{R}^{2}$ range: $0.58-0.78$ ). The association between adjusted BMC, either measured subregionally or across the whole vertebral area, and vertebral failure load, was increased for the subgroup of specimens with identified "low aBMD", compared to those with "high aBMD", particularly in the anterior subregion where the adjusted $R^{2}$ differed by 0.44 .

The relative contribution of $\mathrm{BMC}$ measured in vertebral subregions to ultimate failure load is greater among specimens with lower aBMD, compared to those with higher aBMD, particularly in the anterior subregion of the vertebral body.

Keywords: subregion; bone mineral density; failure; DXA; vertebra 


\section{Introduction}

It is well established that bone microstructure and quantity are distributed heterogeneously throughout the vertebral body [1-8] and that variance in these distributions influences bone strength $[9-11,7,8]$, and responses to bisphosphonate therapy [12]. The intra-vertebral distribution of bone microstructure and quantity is therefore likely to have important implications for the mechanisms underlying vertebral fracture [13-16]. We have previously undertaken research to establish the precision and accuracy of a subregional bone mineral density (srBMD) method $[2,3,6]$ and its application in the clinical setting $[14,17]$. Comparing differences in srBMD values between individuals may be confounded by the variability in their bone size. However, this variability can be overcome by comparing the ratios of srBMD values (that is the ratio of one subregion to another within the same vertebral body) among individuals, rather than their absolute aBMD values. Using ratios of srBMD to compare between individuals removes the potentially confounding influence of differences in the sizes of the vertebrae, and as such of the subregions, between individuals. Our preliminary clinical data suggest that there is little difference in the ratios of srBMD between individuals with normal areal bone mineral density (aBMD) and those with primary osteoporosis, inferring there is a general, homogeneous vertebral bone loss associated with primary osteoporosis, not confined to a preferential subregional area within the vertebra. However, we have observed selective subregional bone loss among individuals with glucocorticoid-induced osteoporosis (GIO) [17], which may suggest selective subregional vertebral bone loss in specific disease states, such as in GIO, or a change in selectivity in individuals with advanced disease state. It is warranted, therefore, to explore the contribution of subregional vertebral bone mineral measures in predicting vertebral failure in differing states of vertebral bone loss.

While generally less precise than postero-anterior (PA)-projection scans, bone mineral measurements derived from lateral-projection scans may have the potential to improve fracture 
prediction estimates. Despite the general low acceptance of lateral-projection scanning, largely attributed to poorer precision and relative lack of reference data, a web of evidence points to the potential clinical utility of bone mineral data derived from lateral-projection scans compared to PAprojection scans, including a stronger association with vertebral failure strength $[18,19]$, superior diagnostic sensitivity $[20,21,14,22,23]$ and greater sensitivity to changes in bone mineral measurements with therapy [24-27] or ageing [21]. However, despite greater sensitivity to changes in bone mineral measurements with therapy, Blake et al [24] concluded that PA-projection measures are the most suitable for longitudinal studies. Further, Bjarnason et al [28] also identified that while lateral projection scanning had a diagnostic advantage in vitro, consistent with more recent data $[19,18]$, this diagnostic advantage was not sustained in situ due to a lower ratio of bone to soft tissue in the lateral projection mode. Ultimately, the choice of scanning projection revolves around the balance between sensitivity in fracture prediction on the one hand and measurement precision on the other. For example, an added advantage of the lateral-projection approach is the ability to measure aBMD in vertebral subregions $[2,3]$, which may improve measurement specificity for DXA, and ultimately improve fracture risk prediction [3], particularly in patients with GIO [17]. In this study we have chosen to explore whether sensitivity can be enhanced by using subregional bone mineral measures derived from lateral projection scans. If successful, this methodological development could lead to a re-appraisal of the diagnostic utility of lateral spine scanning.

The aim of this study was to examine the relationship between subregional bone mineral content (BMC) derived from lateral-projection DXA from human cadaveric spine specimens and vertebral failure strength determined experimentally. We hypothesised that, in vertebrae with more advanced bone loss, subregional BMC measurements would more strongly predict vertebral body strength compared to specimens with less advanced bone loss. 


\section{Materials and Methods}

\section{$\underline{2.1 \quad \text { Specimens }}$}

The imaging and mechanical testing procedures used in this study have been reported in detail elsewhere $[2,3,18]$, and are described briefly below. Twenty-five vertebral bodies (L2-L4), derived from a set of 10 embalmed and 5 fresh-frozen cadaver spines (7 male, 8 female; mean (SD) age at death 78.2 (9.6) years), were available for analysis in this study (Table 1). The 10 embalmed donor cadavers were fixed with $20-40 \mathrm{~L}$ of embalming fluid ( $55 \%$ ethanol, $5 \%$ formaldehyde, $5 \%$ phenol, $20 \%$ propylene glycol and $15 \%$ water) and stored at $4 \mathrm{C}$ for 3 months prior to harvesting of the spine. Previous investigators have found no effect of formalin fixation on vertebral BMD estimations by DXA on embalmed specimens compared to fresh specimens [29]. Therefore, data derived from fresh and embalmed specimens were pooled when examining vertebral bone mineral distributions across the sample (see data analysis section 2.5). The 5 fresh-frozen specimens were frozen at $-17^{\circ} \mathrm{C}$. In all specimens, the ribs and ilia were removed from the spine segments leaving intact vertebral bodies and connective tissues. Embalmed specimens were sealed in water-tight shrink-wrap thermoplastic while fresh-frozen specimens remained in gauze wrap. Prior to any scanning, lateral radiographs were acquired from each specimen to screen for vertebral fractures and any other overt bone pathology and to verify vertebral levels in conjunction with a PA-projection DXA image. Fresh-frozen specimens were scanned in a frozen state, removed from the freezer just before the commencement of scanning. The cadavers used in this study were donated by the next-of-kin of the deceased for use in medical research under the terms and conditions contained within the Anatomy Act of South Australia. The specific terms that apply to this study are that the research be approved by the institutional research committees. Approval to use the specimens for research purposes was granted by the Human Research Ethics Committee at the Royal Adelaide Hospital, South Australia, and Curtin University, Western Australia.

Each specimen was scanned using DXA and subsequently mechanically tested to failure. 


\subsection{Dual energy X-ray absorptiometry (DXA)}

All scanning was performed using a Hologic (Hologic Inc., Bedford, MA; USA) QDR4500A fan beam densitometer, with a spatial resolution of $1.0 \mathrm{~mm}$, running operating software version 9.10D. Spine samples were placed supine in a water bath $(270180150 \mathrm{~mm})$ of tap water to a depth of $18 \mathrm{~cm}$ to simulate soft tissue composition in vivo. This procedure has been used in previous ex vivo studies with validity and reliability established for both lumbar and thoracic vertebrae [30,19,31-33]. A matched PA-supine lateral scan pair was acquired on each specimen using the array scanning mode. Each lateral scan was performed with a fixed scan length of $15.32 \mathrm{~cm}$ and width of $14.47 \mathrm{~cm}$ and pixel size of $1.007 \mathrm{~mm}$. At the completion of the lateral scan, both a standard analysis and a customised subregional analysis were performed. Areal BMD was calculated for the whole vertebral body area (defined as region of interest (ROI) 1) and within six subregions: three oriented sagittally (ROIs 2-4) and three transversely (ROls 5-7) (Figure 1). Subregions were created manually by modifying the regions of interest during the analysis phase. The whole vertebral area (ROI 1 ) was defined by the four corners of the vertebra of interest from the lateral DXA image, including the vertebral endplate and excluding the posterior elements. Overt osteophytes were excluded from the ROls and deleted from the bone map manually, in agreement with previous work $[31,34,35]$. The size and shape of ROI 1 was defined according to the morphology of the vertebral body. The endplates defined the superior and inferior margins, the anterior border of the centrum defined the anterior margin, while the posterior margin was defined by the junction between the vertebral centrum and pedicle of the posterior elements. Subregions 2-4 formed equal thirds in the area of ROI 1, oriented sagittally. Subregions 5-7 formed equal thirds in area of ROI 1, oriented transversely. Our pilot data demonstrate good to fair short-term precision of this protocol when applied at L2 and L3 ex vivo (\% CV range 1.8-6.8\%) [36]. We have also established acceptable precision of the subregional measurement protocol in an in vivo context [6]. 


\subsection{Measurement of vertebral geometry}

Absolute vertebral height was measured by digitising vertebral dimensions visible on the lateral DXA scan. Digital images of the lateral DXA scan were imported into image processing software (Image J version 1.30, National Institute of Health, Maryland, USA). Images were digitised to extract Cartesian $x, y$ coordinates of corners of each vertebral body. After correcting for rotation, $x, y$ coordinates were transformed into real dimensional units $(\mathrm{cm})$ to determine vertebral height.

\subsection{Mechanical testing}

After DXA scanning, the vertebral bodies were mechanically tested to failure. The specimen preparation and testing procedure was performed based on previously published protocols $[37,18,38]$. On both the endplates, a 3mm-thick layer of polymethylmethacrylate (PMMA) was applied, by submerging the vertebra into a curing PMMA mixture. The specimens were soaked for 12 hrs in saline solution at room temperature for rehydration $[19,18]$, before being placed in the mechanical testing machine (Model 800L, TestResources Inc, Shakopee, MN, USA). The uniaxial compression tests were performed between steel platens with a lockable ball joint to ensure planoparallel ends [37]. A saline-soaked gauze was used to keep the specimens moist during the experiment, which was done at room temperature. After preconditioning $(5 \mathrm{cycles}$, at $0.1 \mathrm{~Hz}$, between $150 \mathrm{~N}$ and $350 \mathrm{~N}$, then held at $250 \mathrm{~N}$ for 5 minutes), the specimens were tested in displacement control at a rate of $0.15 \mathrm{~mm} / \mathrm{s}[38,37,18]$. The displacement was measured by a transducer (Linear Variable Differential Transformer) attached to the cross-head. Vertebral body strength ( $F_{\text {ult, }}$ expressed in $\mathrm{kN}$ ) was defined as the peak load during the compressive loading (i.e. the ultimate load). The compressive stiffness $(\mathrm{kN} / \mathrm{mm})$ was also calculated, as the slope of the linear portion of the load-displacement curve, between $25 \%$ and $75 \%$ of the failure load [39]. The 
deformation to failure was calculated from the load-displacement curve, by extrapolating the linear elastic portion back to zero load and expressed as a percentage of the original specimen height.

\section{$\underline{2.5 \quad \text { Data analysis }}$}

Descriptive statistics were used to summarise specimen densitometric characteristics and mechanical properties. Mechanical properties were compared between embalmed and fresh-frozen specimens using a one-way ANOVA, including models where parameters were adjusted for vertebral height. A repeated measures ANOVA was performed to evaluate differences in aBMD and BMC between subregions, in keeping with earlier work $[30,14,3,17,2]$. Given this was a within-specimen analysis and that fixation does not influence DXA-measured BMD [29], data from embalmed and fresh-frozen specimens were pooled in the ANOVA models, as performed previously [2]. While there were seven regions of interest (ROIs), the within-subject factor (ROI) was set $a$ priori at $k=4$ to ensure that overlapping subregions were not compared post hoc. That is, sagitally-oriented subregions were not compared with transversely oriented subregions in the same ANOVA model. Therefore, the first ANOVA model included ROIs 1-4 and the second ANOVA model included ROIs 1 and 5-7. Bonferroni adjustments were applied to pairwise comparisons to account for multiple tests. Multivariate regression models were used to quantify the association between ultimate vertebral failure load (dependent variable) and BMC (predictor variable), adjusted for vertebral height and specimen fixation status. Vertebral height was included in the model as an index of vertebral size, as it is a relatively simple measurement and can be directly measured from DXA scans [3] and has been used previously in multiple regression models for this purpose [40]. Due to the inclusion of an index of vertebral size in the regression models, $\mathrm{BMC}$, rather than $\mathrm{aBMD}$, was used as the predictor variable. While formalin fixation does not appear to systematically affect bone mineral measurements, it has been shown to influence compressive strength of vertebral bone [29]. Hence, specimen fixation status was included as a nominal variable in multiple regression models. Multivariate models were 
run for a pooled sample of 25 vertebrae and for two sub-groups of vertebrae categorised as "low aBMD $(n=13)$ " and "high aBMD $(n=12)$ ", based on a median split of lateral-projection aBMD values of the ROI 1 at $0.458 \mathrm{~g} / \mathrm{cm}^{2}$. The aBMD data calculated for ROI 1 were normally distributed. For all regression models, adjusted $\mathrm{R}^{2}$ values were used as the index to quantify strength of association. Data were analysed using IBM SPSS Statistics 19, IBM Corporation.

\section{Results}

\subsection{Descriptive characteristics}

Standard, whole vertebral aBMD was not significantly different between 15 embalmed and 10 freshfrozen lumbar vertebrae in either the PA [mean (SD): $0.80(0.17) \mathrm{g} / \mathrm{cm}^{2}$ and $0.90(0.14) \mathrm{g} / \mathrm{cm}^{2}$, respectively; mean difference $\left.[95 \% \mathrm{Cl}]-0.1[-0.23,0.04] \mathrm{g} / \mathrm{cm}^{2}\right]$ or lateral projections $[0.47(0.12)$ $\mathrm{g} / \mathrm{cm}^{2}$ and $0.54(0.11) \mathrm{g} / \mathrm{cm}^{2}$, respectively; $\left.-0.07[-0.16,0.03] \mathrm{g} / \mathrm{cm}^{2}\right]$. A similar finding was observed for whole vertebral BMC in the PA $[12.6(4.0) \mathrm{g}$ and $14.0(4.0) \mathrm{g}$, respectively; $-1.5[-4.9,1.9] \mathrm{g})$ and lateral (4.8 (2.1) g and $5.6(1.8)$ g, respectively; -0.8 [-2.5, 0.9] g] projections.

\subsection{Subregional $\mathrm{aBMD}$ and $\mathrm{BMC}$ profiles}

A main effect for ROI was observed when comparing $\mathrm{aBMD}$ and $\mathrm{BMC}$ between sagittal and transverse ROIs $(p<0.0001)$. In the sagittal ROI analysis, a significant difference was observed between all ROls for aBMD ( $\leq \leq 0.004$; Figure 2 ) and BMC ( $<<0.0001$; Figure 3$)$. The highest aBMD was observed in the posterior subregion (ROI 2), while the lowest aBMD was observed in the anterior subregion (ROI 4). When considering the intra-vertebral subregions only, the same distribution pattern was observed for BMC profiles. In the transverse ROI analysis, a significant difference was observed between all ROls for aBMD ( $p \leq 0.006$; Figure 2), other than between whole (ROI 1$)$ and superior (ROI 5) regions ( $p=0.11)$. For $B M C$, a significant difference was observed between all subregions ( $p \leq 0.0001$; Figure 3), other than between the superior (ROI 5) and inferior ( $R O I 7)$ 
subregions. The highest aBMD was observed in the inferior subregion (ROI 7), while the lowest aBMD was observed in the central subregion (ROI 6).

\subsection{BMC as a predictor of ultimate vertebral failure}

Table 2 provides a summary of mechanical parameters measured. Mean ultimate vertebral failure load $\left(\mathrm{F}_{\text {ult }}\right)$ and vertebral height-adjusted mean ultimate failure load did not exhibit statistically significant differences between embalmed and fresh-frozen specimens. Mean vertebral stiffness was significantly higher and mean relative ultimate deformation significantly lower, in embalmed specimens.

Multivariate regression models with adjusted BMC as the predictor, explained a substantial proportion of variance in ultimate vertebral load where BMC was measured over the whole vertebral area in lateral projection (ROI 1; adjusted $\mathrm{R}^{2}: 0.84$ ) and across the six subregions (ROIs 2-7) (adjusted $\mathrm{R}^{2}$ range: $0.58-0.80 ; \mathrm{p}<0.0001$ ). $\mathrm{BMC}$ derived from lateral-projection $\mathrm{DXA}$, measured across the whole vertebral area (ROI 1) or within a subregion (ROIs 2-7), accounted for a substantially greater proportion of variance in ultimate load, compared to BMC derived from PA-projection (Table 2). Both vertebral height and fixation status remained significant co-variates across most multivariate models, other than the high aBMD subgroup where vertebral height was not a significant predictor of failure load (Table 3).

Across all the specimens, subregional BMC (ROIs 2-7) was not a stronger predictor of ultimate vertebral failure load than BMC measured across the whole vertebral area (ROI 1) (Table 3). However, when analysed in aBMD subgroups, subregional BMC accounted for a greater proportion of variance in ultimate vertebral failure load in the low aBMD subgroup, compared to the high aBMD subgroup (mean increase in variance explained: 18\%). This was particularly noticeable for the change 
in adjusted $R^{2}$ at ROIs 4,6 , and 7 , where the adjusted $R^{2}$ values increased by $0.44,0.15$, and 0.24 , respectively, compared to the high aBMD group (Figure 4). Within the low aBMD subgroup, BMC measured at ROIs 4 and 7 explained a greater proportion of variance in ultimate vertebral load, than $\mathrm{BMC}$ measured at ROI 1, although not by a clinically meaningful amount. Similar to the multivariate regression models using the whole dataset, measures of BMC derived from lateral projection DXA, either whole vertebral (ROI 1) or subregional (ROIs 2-7), explained a greater proportion of variance in ultimate load than BMC derived from PA-projection DXA (Table 3).

\section{Discussion}

Consistent with previous investigations, this study confirms that vertebral aBMD and BMC measured by lateral-projection DXA vary within the vertebral body $[13,22,3]$. In accordance with existing literature $[33,18,19,41], \mathrm{BMC}$ was found to be a strong predictor of ultimate vertebral failure load, and a substantially greater proportion of variance in failure load could be explained by BMC when measured using lateral-projection DXA, compared with PA-projection DXA (e.g., $\mathrm{R}^{2}=0.86$ vs. 0.57 ). This finding supports the potential for broader application of lateral-projection DXA in clinical settings. Moreover, when subgrouping our cohort into vertebrae with lower and higher aBMD, variations in vertebral failure load were better explained by adjusted $\mathrm{BMC}$ values for specimens with lower aBMD. In particular for subregional BMC measures, vertebrae with lower aBMD exhibited stronger correlations $\left(R^{2}\right.$ up to 0.88$)$ with failure load, compared to vertebrae with higher aBMD $\left(R^{2}\right.$ up to 0.72 ). Although, when compared to lateral BMC measures derived from the whole vertebral body $\left(R^{2}=0.84\right)$, the increase in $R^{2}$ value due to subregional $B M C$ measures unlikely represents a clinically-meaningful improvement in magnitude, our data highlight the potential biological and biomechanical role of subregional BMC in influencing vertebral fragility in circumstances of low aBMD. 
Differences in $\mathrm{aBMD}$ and $\mathrm{BMC}$ between subregions demonstrated in this study are comparable to those we have observed in clinical $[6,17]$ and ex vivo [30] studies previously. It is most likely that the patterns of distribution of subregional bone mineral properties influence the mechanical behaviour of the vertebral body, particularly the relative distributions between subregions. For example, DXAderived $\mathrm{ABMD}$ and $\mathrm{BMC}$ are lower in the anterior and central subregions, compared to other regions such as the posterior region and regions adjacent to the endplates. This observation is likely due to the relatively lower amount of bone present in these regions, particularly in the central region (ROI 6) as shown in our previous studies including using pQCT [22,3] and may point to a mechanism underlying vertebral anterior wedge fractures [42]. The profiles of subregional aBMD and BMC appear to be consistent across ex vivo studies [2,14], consistent across imaging modalities [3], and consistent between individuals with normal $\mathrm{aBMD}$ and $\mathrm{BMC}$ and those with primary osteoporosis, suggesting non-selective vertebral bone loss. We demonstrated recently that the vertebral subregional aBMD distribution profile appears to differ among individuals with GIO [17], suggesting selective vertebral bone loss in specific vertebral subregions, particularly ROI 4. Although this finding needs verification in larger studies with multiple patient groups, it does provide a rationale that subregional bone distribution measured by DXA may have important biological significance to vertebral strength in specific patient groups, and/or in vertebrae with advanced bone loss. Indeed, the results from this study highlight that subregional $\mathrm{BMC}$ is a stronger predictor of vertebral failure load in a group of specimens presenting with lower aBMD values compared to specimens with high aBMD. This finding was particularly evident in the anterior subregion, where a further $44 \%$ of variance in failure load was explained in samples with low aBMD compared to high aBMD. The strong relationship between $\mathrm{BMC}$ measured in the anterior subregion with vertebral failure load in specimens with low aBMD is likely related to the extent of intervertebral disc degeneration. Earlier studies have demonstrated an association between disc degeneration and relative increased bone loss in the anterior region of the vertebral body $[5,42]$, which is likely to lead to increased propensity 
to vertebral body failure. We are unable to speculate further on these associations as we have no data regarding disc degeneration in our specimen sample. However, this represents an important consideration for future research.

While we acknowledge that the proportion of variance explained by subregional measures of BMC (ROI 4) was only up to $4 \%$ greater than that explained by $\mathrm{BMC}$ measured from the whole vertebral body, the finding is consistent with previous work [9]. Moreover, the difference in $\mathrm{R}^{2}$ values between multivariate models using high aBMD samples compared to low aBMD samples was substantially greater for the subregions $(0.03-0.44)$, compared to the whole vertebral BMC $(0.11)$, demonstrating an increasing contribution of subregional BMC to influencing vertebral failure as bone loss progresses. Here, the relative importance of subregional bone mineral measures may be related to progressively selective bone loss [43], influenced for example, through intervertebral disc degeneration [5].

Our data suggest that BMC measured in the superior and inferior ROIs (ROIs 5 and 7) has a more substantial role in determining vertebral strength in uniaxial compressive testing, compared to BMC derived from the central $\mathrm{ROI}(\mathrm{ROI} 6)$, pointing to a critical role of the bone in the superior and inferior zones of the vertebral body in modulating vertebral failure in compression. This observation was consistent across specimens with high and low aBMD and concurs with results from CT-based finite element modelling by Eswaran et al [44] and Homminga et al [45].

This study had several important strengths. First, although the likely importance of subregional bone mineral properties in modulating vertebral failure load has been noted previously for BMD [9] and bone volume fraction [10] particularly in the anterior region, measurements of bone mineral 
properties in this area using DXA and linking them to failure load have not previously been explored. Second, we mechanically tested a large sample of vertebrae which allowed us to undertake subgroup analyses based on a median split of aBMD values. Third, we examined the association between failure load and BMC using multivariate models, accounting for the potentially confounding influence of specimen fixation and vertebral size. Moreover, the standardised regression coefficients reported in this study are comparable to those reported previously by Edmondston et al [40]. Some important limitations should be considered in the interpretation of our results. First, the loading condition we applied to the vertebrae is not completely representative of physiologic conditions. Although compression in inferior-superior direction is the predominant loading direction in upright stance on the lumbar spine [46], the experimental protocol used in the study does not account for other forces or moments the vertebrae would experience in vivo, such as bending moments. More importantly, the significant role of the intervertebral disc in transferring load across the vertebral body is not considered in our protocol, nor the influence of disc degeneration on load transmission across the vertebral body and the likely secondary consequences of altered load transmission on subregional bone mineral properties $[5,42,47]$. Second, we chose to employ DXA as the imaging modality in this study owing to its ubiquitous use in the clinical environment. Consequently, our inferences regarding the relationship between $\mathrm{BMC}$ and load are limited to composite measures of trabecular and cortical bone and do not enable us to explore the important contributions of these bone components independently [18], or the contribution of subregional bone micro-architecture to mechanical behaviour [39]. Further, matched PA-lateral scans are only available with Hologic densitometers. While other manufacturers enable acquisition of lateral-projection scans, a decubitus position is generally required which is associated with unacceptably low precision and substantially longer operator time. Third, the experimental protocol involving a water bath to acquire DXA scans does not truly correspond with the clinical environment where scan artefacts attributed to variance in soft tissue composition of the trunk and arterial calcification are commonplace. Finally, when considering our pooled sample of 15 donors, the mean PA-projection derived aBMD values 
represent that of an aged population with increased risk of fracture, reflecting osteoporosis (males: mean[sd] aBMD $=0.781[0.142] \mathrm{g} / \mathrm{cm}^{2}$; females: $\left.0.725[0.185] \mathrm{g} / \mathrm{cm}^{2}\right)$, based on the aBMD values reported by Henry et al [48] (average values below -2.5 SD). When sub-grouped, donors in the low aBMD fell in the osteoporosis range (PA aBMD values below $-2.5 \mathrm{SD}$ ), while those in the high aBMD fell in the osteopenic range (PA aBMD values between -1 and $-2.5 \mathrm{SD}$ ). This, together with the advanced age, suggests that subjects sampled in our study might generally represent a subgroup with an increased risk of fracture compared to the normal population. Importantly, these estimates have been calculated by adjusting our raw data for scanner type [49] and for data derived from exsitu scanning where values are recommended to be lowered by $10 \%$ to be compared to in-situ scanning [50], consistent with an approach we have reported previously [18].

Given the expanding web of knowledge surrounding the potential biological significance of measuring subregional bone mineral properties with lateral-projection DXA, it will now be important to verify lab-based findings with further clinical studies to elucidate changes in subregional bone properties over time and in response to therapies. It will also be important to verify the associations between subregional bone mineral properties and mechanical behaviour of whole vertebral bone using experimental loading conditions which more closely replicate physiologic loading. Ultimately, clinical studies that compare fracture prediction by different DXA modalities will be important.

\section{Conclusion}

BMC measured from lateral-projection DXA, either across the whole vertebral area or within vertebral subregions, is a stronger predictor of failure load than BMC measured from PA-projection DXA. The relative contribution of BMC measured in vertebral subregions to ultimate failure load is substantially greater among specimens with lower global aBMD, particularly in the anterior 
subregion of the vertebral body, as opposed to those with high aBMD. In specimens with low aBMD, $\mathrm{BMC}$ measured from the anterior subregion accounts for a slightly greater proportion of variance in vertebral failure load than a measure of whole vertebral BMC.

\section{Acknowledgements}

The authors respectfully acknowledge the late Susan Kantor, Senior Bone Densitometrist, who contributed substantially to data collection and analysis and Professor Nicola (Nick) Fazzalari who contributed to earlier studies leading to this work. Dr Andrew Briggs was supported, in part, by a fellowship awarded by the Australian National Health and Medical Research Council (NHMRC). Funding for this study was provided by the NHMRC, Scoliosis Research Society (USA), and Arthritis Australia. In kind support was provided by the Bone Densitometry Unit (University of Melbourne Department of Medicine, Royal Melbourne Hospital), SA Pathology and the Ray Last Anatomy Laboratory, School of Medical Sciences, The University of Adelaide.

\section{Conflict of interest disclosure statement}

The authors declare no conflicts of interest

\section{References}

1. Banse X, Devogelaer JP, Munting E, Delloye C, Cornu O, Grynpas M (2001) Inhomogeneity of human vertebral cancellous bone: Systematic density and structure patterns inside the vertebral body. Bone 28: 563-571 
2. Briggs AM, Perilli E, Parkinson IH, Kantor S, Wrigley TV, Fazzalari NL, Wark JD (2012) Measurement of subregional vertebral bone mineral density in vitro using lateral projection dual-energy X-ray absorptiometry: validation with peripheral quantitative computed tomography. J Bone Miner Metab 30: $222-231$

3. Briggs AM, Perilli E, Parkinson IH, Wrigley TV, Fazzalari NL, Kantor S, Wark JD (2010) Novel assessment of subregional bone mineral density using DXA and pQCT, and subregional microarchitecture using micro-CT in whole human vertebrae: Applications, methods, and correspondence between technologies. J Clin Densitom 13: 161-174

4. Cvijanovic O, Bobinac D, Zoricic S, Ostojic Z, Maric I, Crncevic-Orlic Z, Kristofic I, Ostojic L (2004) Age and region dependent changes in human lumbar vertebral bone. A histomorphometric study. Spine 24: $2370-2375$

5. Simpson EK, Parkinson IH, Manthey B, Fazzalari NL (2001) Intervertebral disc disorganisation is related to trabecular bone architecture in the lumbar spine. J Bone Miner Res 16: 681-687

6. Briggs AM, Wark JD, Kantor S, Teh R, Greig AM, Fazzalari NL, Bennell KL (2005) In vivo intra-rater and inter-rater precision of measuring apparent bone mineral density in vertebral subregions using supine lateral dual- energy x-ray absorptiometry (DXA). J Clin Densitom 8: 314-319

7. Hulme PA, Boyd SK, Ferguson SJ (2007) Regional variation in vertebral bone morphology and its contribution to vertebral fracture strength. Bone 41: 946-957

8. Hussein Al, Morgan EF (2013) The effect of intravertebral heterogeneity in microstructure on vertebral strength and failure patterns. Osteoporos Int 24: 979-989

9. McCubbery DA, Cody DD, Peterson EL, Kuhn JL, Flynn MJ, Goldstein SA (1995) Static and fatigue failure properties of thoracic and lumbar vertebral bodies and their relation to regional density. J Biomech 28: 891-899 
10. Kim DG, Hunt CA, Zauel R, Fyhrie DP, Yeni YN (2007) The effect of regional variations of the trabecular bone properties on the compressive strength of human vertebral bodies. Ann Biomed Engin 35: 1907-1913

11. Yeni YN, Zinno MJ, Yerramshetty JS, Zauel R, Fyhrie DP (2011) Variability of trabecular microstructure is age-, gender-, race- and anatomic site-dependent and affects stiffness and stress distribution properties of human vertebral cancellous bone. Bone 49: 886-894

12. Engelke K, Fuerst T, Dasic G, Davies RY, Genant HK (2010) Regional distribution of spine and hip QCT BMD responses after one year of once-monthly ibandronate in postmenopausal osteoporosis. Bone 46: 1626-1632

13. Briggs AM, Greig AM, Wark JD (2007) The vertebral fracture cascade in osteoporosis. A review of aetiopathogenesis. Osteoporos Int 18: 575-584

14. Briggs AM, Wark JD, Greig AM, Fazzalari NL, Kantor S, Bennell KL (2009) Subregional bone mineral density measurement in the lumbar spine using DXA: Potential for the application to osteoporosis and vertebral fractures. In: Mattingly BE, Pillare AC (eds) Osteoporosis: Etiology, Diagnosis and Treatment. Nova Publishers, New York, pp 1-50

15. Perilli E, Baleani M, Ohman C, Fognani R, Baruffaldi F, Viceconti M (2008) Dependence of mechanical compressive strength on local variations in microarchitecture in cancellous bone of proximal human femur. J Biomech 41: 438-446

16. Nazarian A, Stauber M, Zurakowski D, Snyder BD, Muller R (2006) The interaction of microstructure and volume fraction in predicting failure in cancellous bone. Bone 39: 1196-1202 17. Manning LI, Briggs AM, Van Doornum S, Kale A, Kantor S, Wark JD (2013) Glucocorticoid-induced bone loss is associated with abnormal intravertebral areal bone mineral density distribution. Int J Endocrinol 2013: 768579 
18. Perilli E, Briggs AM, Kantor S, Codrington J, Wark JD, Parkinson IH, Fazzalari NL (2012) Failure strength of human vertebrae: prediction using bone mineral density measured by DXA and bone volume by micro-CT. Bone 50: 1416-1425

19. Burklein D, Lochmuller EM, Kuhn V, Grimm J, Barkmann R, Muller R, Eckstein F (2001) Correlation of thoracic and lumbar vertebral failure loads with in situ vs. ex situ dual energy X-ray absorptiometry. J Biomech 34: 579-587

20. Jergas M, Genant HK (1997) Lateral dual X-ray absorptiometry of the lumbar spine: Current status. Bone 20: 311-314

21. Zmuda JM, Cauley JA, Glynn NW, Finkelstein JS (2000) Posterior-anterior and lateral dual-energy x-ray absorptiometry for the assessment of vertebral osteoporosis and bone loss among older men. J Bone Miner Res 15: 1417-1424

22. Briggs AM, O'Sullivan PB, Foulner D, Wark JD (2012) Vertebral bone mineral measures and psychological wellbeing among individuals with modic changes. Clin Med Insights Case Rep 5: 35-41

23. Briggs AM, Straker LM, Burnett AF, Wark JD (2012) Chronic low back pain is associated with reduced vertebral bone mineral measures in community-dwelling adults. BMC Musculoskelet Disord 13: 49

24. Blake GM, Herd RJM, Fogelman I (1996) A longitudinal study of supine lateral DXA of the lumbar spine: A comparison with posteroanterior spine, hip and total-body DXA. Osteoporos Int 6: 462-470 25. Reid IR, Evans MC, Stapleton J (1992) Lateral spine densitometry is a more sensitive indicator of glucocorticoid-induced bone loss. J Bone Miner Res 7: 1221-1225

26. Finkelstein JS, Klibanski A, Arnold AL, Toth TL, Hornstein MD, Neer RM (1998) Prevention of estrogen deficiency-related bone loss with human parathyroid hormone-(1-34) - A randomized controlled trial. J Am Med Assoc 280: 1067-1073 
27. Finkelstein JS, Wyland JJ, Leder BZ, Burnett-Bowie S-AM, Lee H, Jueppner H, Neer RM (2009)

Effects of Teriparatide Retreatment in Osteoporotic Men and Women. J Clin Endocrinol Metab 94: 2495-2501

28. Bjarnason K, Hassager C, Svendsen OL, Christiansen C (1996) Anteroposterior and lateral spinal DXA for the assessment of vertebral body strength: Comparison with hip and forearm measurement. Osteoporos Int 6: 37-42

29. Edmondston SJ, Singer KP, Day RE, Breidahl PD, Price RI (1994) Formalin fixation effects on vertebral bone density and failure mechanics. An in-vitro study of human and sheep vertebrae. Clin Biomech 9: 175-179

30. Briggs AM, Wark JD, Kantor S, Fazzalari NL, Greig AM, Bennell KL (2006) Bone mineral density distribution in thoracic and lumbar vertebrae: An ex vivo study using dual energy X-ray absorptiometry. Bone 38: 286-288

31. Edmondston SJ, Singer KP, Price RI, BreidahI PD (1993) Accuracy of lateral dual-energy x-ray absorptiometry for the determination of bone mineral content in the thoracic and lumbar spine: an in vitro study. Br J Radiol 66: 309-313

32. Kaymakci B, Wark JD (1994) Precise accurate mineral measurements of excised sheep bones using X-Ray densitometry. Bone Miner 25: 231-246

33. Singer K, Edmondston S, Day R, Breidahl P, Price R (1995) Prediction of thoracic and lumbar vertebral body compressive strength. Correlations with bone mineral density and vertebral region. Bone 17: 167-174

34. Sran MM, Khan KM, Keiver K, Chew JB, McKay HA, Oxland TR (2005) Accuracy of DXA scanning of the thoracic spine: cadaveric studies comparing BMC, areal BMD and geometric estimates of volumetric BMD against ash weight and CT measures of bone volume. Eur Spine J 14: 971-976 
35. Jones G, White C, Nguyen T, Sambrook PN, Kelly PJ, Eisman JA (1996) Prevalent vertebral deformities: relationship to bone mineral density and spinal osteophytosis in elderly men and women. Osteoporos Int 6: 233-239

36. Briggs AM (2006) Pathomechanics of spinal osteoporosis: Subregional bone mineral density and physiologic loading. PhD thesis, The University of Melbourne, Melbourne

37. Kopperdahl DL, Pearlman JL, Keaveny TM (2000) Biomechanical consequences of an isolated overload on the human vertebral body. J Orthop Res 18: 685-690

38. Fields AJ, Eswaran SK, Jekir MG, Keaveny TM (2009) Role of trabecular microarchitecture in whole-vertebral body biomechanical behavior. J Bone Miner Res 24: 1523-1530

39. Wegrzyn J, Roux JP, Arlot ME, Boutroy S, Vilayphiou N, Guyen O, Delmas PD, Chapurlat R, Bouxsein ML (2010) Role of trabecular microarchitecture and its heterogeneity parameters in the mechanical behavior of ex vivo human L3 vertebrae. J Bone Miner Res 25: 2324-2331

40. Edmondston SJ, Singer KP, Day RE, Price RI, BreidahI PD (1997) Ex vivo estimation of thoracolumbar vertebral body compressive strength: The relative contributions of bone densitometry and vertebral morphometry. Osteoporos Int 7: 142-148

41. Myers BS, Arbogast KB, Lobaugh B, Harper KD, Richardson WJ, Drezner MK (1994) Improved assessment of lumbar vertebral body strength using supine lateral dual-energy X-ray absorptiometry. J Bone Miner Res 9: 687-693

42. Pollintine $P$, Dolan $P$, Tobias JH, Adams MA (2004) Intervertebral disc degeneration can lead to "stress-shielding" of the anterior vertebral body - A cause of osteoporotic vertebral fracture? Spine 29: $774-782$

43. Fields AJ, Nawathe S, Eswaran SK, Jekir MG, Adams MF, Papadopoulos P, Keaveny TM (2012) Vertebral fragility and structural redundancy. J Bone Miner Res 27: 2152-2158 
44. Eswaran S, Gupta A, Adams MF, Keaveny TM (2006) Cortical and trabecular load sharing in the human vertebral body. J Bone Miner Res 21: 307-314

45. Homminga J, Weinans H, Gowin W, Felsenberg D, Huiskes R (2001) Osteoporosis changes the amount of vertebral trabecular bone at risk of fracture but not the vertebral load distribution. Spine 24: $1555-1561$

46. Briggs AM, Wrigley TV, Dieen JHv, Phillips B, Lo SK, Greig AM, Bennell KL (2006) The effect of osteoporotic vertebral fracture on predicted spinal loads in vivo. Eur Spine J 15: 1785-1795

47. Hulme PA, Boyd SK, Ferguson SJ (2007) Regional variation in vertebral bone morphology and its contribution to vertebral fracture strength. Bone 41: 946-957

48. Henry MJ, Pasco JA, Pocock NA, Nicholson GC, Kotowicz MA (2004) Reference ranges for bone densitometers adopted Australia-wide: Geelong osteoporosis study. Australas Radiol 48: 473-475

49. Genant HK, Grampp S, Gluer CC, Faulkner KG, Jergas M, Engelke K, Hagiwara S, Van Kuijk C (1994) Universal standardization for dual $\mathrm{x}$-ray absorptiometry: patient and phantom cross-calibration results. J Bone Miner Res 9: 1503-1514

50. Lochmuller EM, Krefting N, Burklein D, Eckstein F (2001) Effect of fixation, soft-tissues, and scan projection on bone mineral measurements with dual energy X-ray absorptiometry (DXA). Calcif Tissue Int 68: 140-145 
Table 1 Summary of specimens used in this study by gender

\begin{tabular}{lcc}
\hline Vertebral level & Embalmed ( $\mathbf{n}=\mathbf{1 0}$ donors) & Fresh-frozen $(\mathbf{n}=\mathbf{5}$ donors) \\
\hline L2 & 4 male, $\mathbf{3}$ female & 2 male, 1 female \\
L3 & 3 male, 5 female & 2 male, 3 female \\
L4 & 0 & 2 female \\
Mean (SD) age at death: yrs & $77.3(10.3)$ & $79.6(8.9)$ \\
\hline
\end{tabular}


difference and 95\% confidence interval $(\mathrm{Cl})$ are provided for the unadjusted difference, and vertebral height-adjusted difference.

\begin{tabular}{lcccc}
\hline Mechanical parameter & Embalmed & Fresh-frozen & Mean difference (95\% Cl) & Adjusted mean difference (95\% Cl) \\
\hline Ultimate load $\left(\mathrm{F}_{\text {ult }}\right)(\mathrm{kN})$ & $5.00(2.28)$ & $3.83(1.40)$ & $1.17(-0.50,2.84)$ & $1.09(-0.62,2.81)$ \\
Stiffness $(\mathrm{kN} / \mathrm{mm})$ & $8.52(3.43)$ & $5.05(1.86)^{\wedge}$ & $3.46(1.00,5.93)^{\wedge}$ & $3.23(0.78,5.69)^{\wedge}$ \\
Relative ultimate deformation $(\%)$ & $2.21(0.43)$ & $3.46(1.08)^{\wedge}$ & $-1.24(-1.88,-0.61)^{\wedge}$ & $\mathrm{n} / \mathrm{a}$ \\
\hline${ }^{\wedge}$ significant difference $(\mathrm{p}<0.05)$ & & & &
\end{tabular}


Table 3: $\quad$ Multivariate regression model results for each vertebral region of interest (ROI), analysed as a pooled data set $(n=25)$ and by low aBMD $(n=13)$ and high aBMD $(n=12)$ subgroups. The proportion of variance in the dependent variable (failure load) explained by the predictor variable (BMC), adjusted for vertebral height and fixation, is expressed as the $\mathrm{R}^{2}$ parameter. Standardised regression coefficients for each component of the multivariate model indicate the relative contribution of each component in explaining the total variance in the dependent variable.

\begin{tabular}{|c|c|c|c|c|c|c|}
\hline \multirow[b]{2}{*}{ Sample } & \multirow[b]{2}{*}{ ROI } & \multirow[b]{2}{*}{$\begin{array}{c}\text { unadjusted } \\
\mathbf{R}^{2}\end{array}$} & \multirow[b]{2}{*}{$\begin{array}{c}\text { adjusted } \\
\mathbf{R}^{2}\end{array}$} & \multicolumn{3}{|c|}{ Standardised regression coefficients } \\
\hline & & & & BMC & $\begin{array}{c}\text { Vert. } \\
\text { height }\end{array}$ & $\begin{array}{l}\text { Fixation } \\
\text { status }^{\mathrm{e}}\end{array}$ \\
\hline \multicolumn{7}{|l|}{ Pooled } \\
\hline & 1 & 0.86 & 0.84 & 1.06 & -0.40 & -0.66 \\
\hline & 2 & 0.81 & 0.78 & 0.97 & -0.28 & -0.62 \\
\hline & 3 & 0.81 & 0.78 & 0.99 & -0.32 & -0.61 \\
\hline & 4 & 0.67 & 0.63 & 0.99 & -0.44 & -0.65 \\
\hline & 5 & 0.79 & 0.76 & 1.01 & -0.34 & -0.67 \\
\hline & 6 & 0.63 & 0.58 & 0.86 & $-0.21^{\mathrm{a}}$ & -0.63 \\
\hline & 7 & 0.82 & 0.8 & 1.02 & -0.41 & -0.54 \\
\hline & PA-projection & 0.57 & 0.51 & 0.73 & $-0.10^{\mathrm{a}}$ & -0.44 \\
\hline \multicolumn{7}{|c|}{ Low aBMD sub-group ${ }^{c}$} \\
\hline & 1 & 0.88 & 0.83 & 0.66 & -0.46 & -0.66 \\
\hline & 2 & 0.83 & 0.77 & 0.63 & -0.44 & -0.63 \\
\hline & 3 & 0.81 & 0.75 & 0.62 & -0.55 & -0.8 \\
\hline & 4 & 0.91 & 0.88 & 0.70 & -0.38 & -0.54 \\
\hline & 5 & 0.83 & 0.77 & 0.63 & -0.48 & -0.65 \\
\hline & 6 & 0.75 & 0.66 & 0.59 & -0.68 & -0.86 \\
\hline & 7 & 0.88 & 0.84 & 0.69 & -0.35 & -0.52 \\
\hline & PA-projection & 0.72 & 0.62 & 0.58 & $-0.31^{a}$ & $-0.44^{a}$ \\
\hline \multicolumn{7}{|c|}{ High aBMD sub-group $^{d}$} \\
\hline & 1 & 0.80 & 0.72 & 0.89 & $-0.35^{\mathrm{a}}$ & -0.73 \\
\hline & 2 & 0.75 & 0.66 & 0.69 & $-0.13^{a}$ & -0.73 \\
\hline & 3 & 0.80 & 0.72 & 0.72 & $-0.13^{a}$ & -0.62 \\
\hline & $4^{b}$ & 0.59 & 0.44 & $0.39^{\mathrm{a}}$ & $0.06^{\mathrm{a}}$ & -0.71 \\
\hline & 5 & 0.74 & 0.65 & 0.69 & $-0.13^{a}$ & -0.77 \\
\hline & 6 & 0.64 & 0.51 & $0.39^{a}$ & $0.16^{\mathrm{a}}$ & -0.68 \\
\hline & 7 & 0.71 & 0.6 & $0.80^{\mathrm{a}}$ & $-0.31^{\mathrm{a}}$ & -0.64 \\
\hline & PA-projection ${ }^{b}$ & 0.58 & 0.42 & $0.26^{a}$ & $0.21^{\mathrm{a}}$ & -0.69 \\
\hline
\end{tabular}
a: variable not a significant predictor in multivariate model $(p>0.05)$
b: multivariate model not significant $(p>0.05)$
c: sub-group defined by median split of aBMD data measured at ROI $1\left(\leq 0.458 \mathrm{~g} / \mathrm{cm}^{2} ; \mathrm{n}=13\right)$
d: sub-group defined by median split of aBMD data measured at ROI $1\left(>0.458 \mathrm{~g} / \mathrm{cm}^{2} ; \mathrm{n}=12\right)$
e: nominal variable defined as $0=$ embalmed and $1=$ fresh-frozen 


\section{Figure legends}

Figure 1: $\quad$ DXA-derived vertebral subregions defined using Hologic software. ROI 1 (whole) was defined by the four corners of the vertebra. ROls 2-4 (posterior, middle, anterior) formed equal thirds in the area of ROI 1, oriented sagittally. ROIs 5-7 (superior, central, inferior) formed equal thirds in area of ROI 1, oriented transversely. Reproduced from the Journal of Clinical Densitometry, Vol 13, Briggs et al., "Novel assessment of subregional bone mineral density using DXA and pQCT, and subregional micro-architecture using micro-CT in whole human vertebrae: Applications, methods, and correspondence between technologies", pp. 161-174, Copyright (2010), with permission from Elsevier Copyright Clearance Center.

Figure 2: $\quad$ Mean areal bone mineral density $\left(\mathrm{aBMD} ; \mathrm{g} / \mathrm{cm}^{2}\right)$ measured across the whole vertebral area (ROI 1) and in the six intra-vertebral subregions (ROIs 2-7). Error bars denote standard error. All pairwise comparisons are Bonferroni corrected $(p \leq 0.006)$ :

$\boldsymbol{a}$ : significantly different to ROI 1 (whole); $\boldsymbol{b}$ : significantly different to ROI 2 (posterior); c: significantly different to ROI 3 (middle); $\boldsymbol{d}$ : significantly different to ROI 4 (anterior); $\boldsymbol{e}$ : significantly different to ROI 5 (superior); $f$ : significantly different to ROI 6 (central); $\boldsymbol{g}$ : significantly different to ROI 7 (inferior).

Figure 3: $\quad$ Mean bone mineral content (BMC; g) measured across the whole vertebral area (ROI 1) and in the six intra-vertebral subregions (ROIs 2-7). Error bars denote standard error. All pairwise comparisons are Bonferroni corrected ( $p \leq 0.0001)$ : $\boldsymbol{a}$ : significantly different to ROI 1 (whole); $\boldsymbol{b}$ : significantly different to ROI 2 (posterior); $\boldsymbol{c}$ : significantly different to ROI 3 (middle); $\boldsymbol{d}$ : significantly different to ROI 4 (anterior); $\boldsymbol{e}$ : significantly different to ROI 5 (superior); $f$ : significantly different to ROI 6 (central); g: significantly different to ROI 7 (inferior). 
Figure 4: $\quad$ Scatterplots illustrating multivariate regression-standardised predicted ultimate vertebral failure load ( $y$ axis) against value of experimentally measured ultimate vertebral failure load ( $x$ axis), in high and low aBMD sub-group analyses for ROI 4 (A and $B$, respectively), ROI 6 (C and $\mathbf{D}$, respectively) and $\mathrm{ROI} 7$ ( $E$ and $\mathrm{F}$, respectively). Each panel displays the unadjusted $R^{2}$ value and the linear regression line of best fit with its $95 \%$ confidence interval (broken lines). 


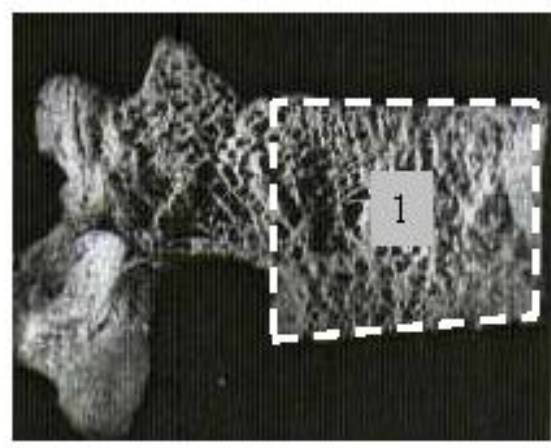

Region 1 - whole area (integrated cortical and trabecular bone) of the vertebral body, defined bythe bur comers of the vertebral body in the sagittal plane.

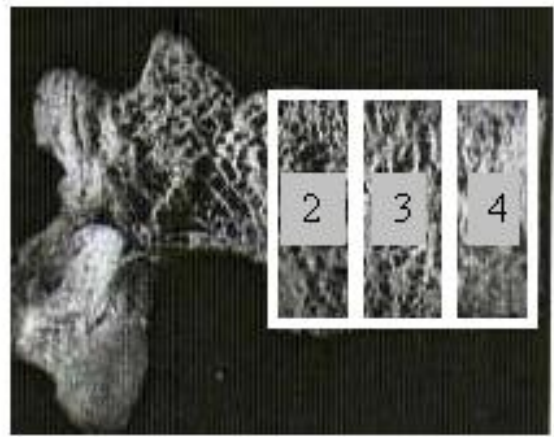

Subregion 2 - posterior thind of ROI 1

Subregion 3 - middle third of ROI 1

Subregion 4 - anteriorthird of ROI 1

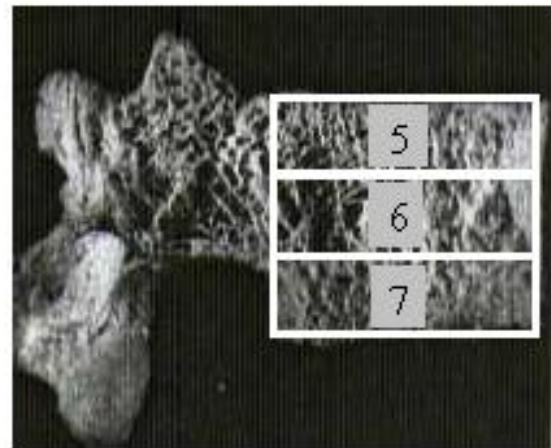

Subregion 5 - supe riorthird of ROI 1

Subregion 6 - central third of ROI 1

Subregion 7 -interiorthird of ROI 1 


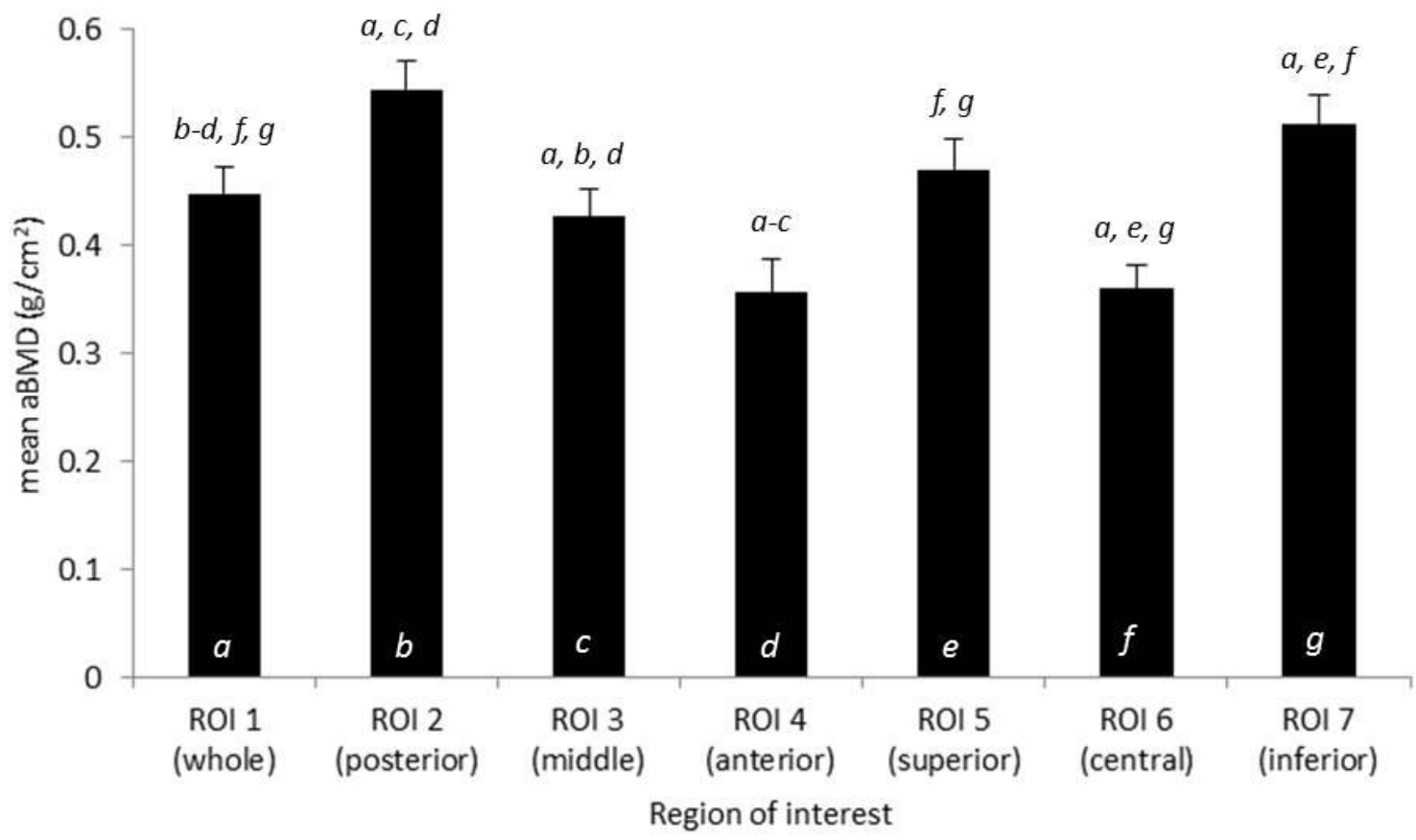




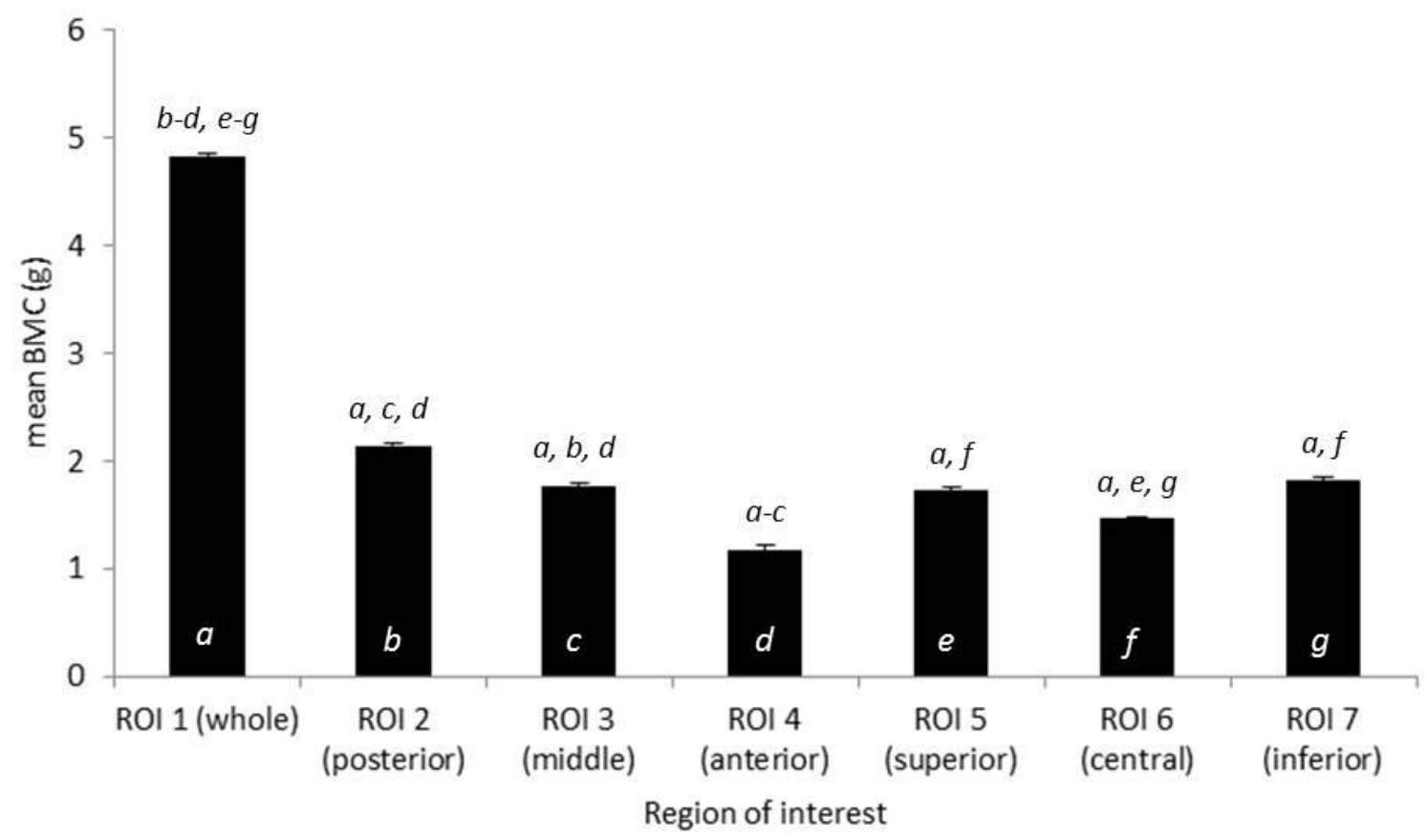



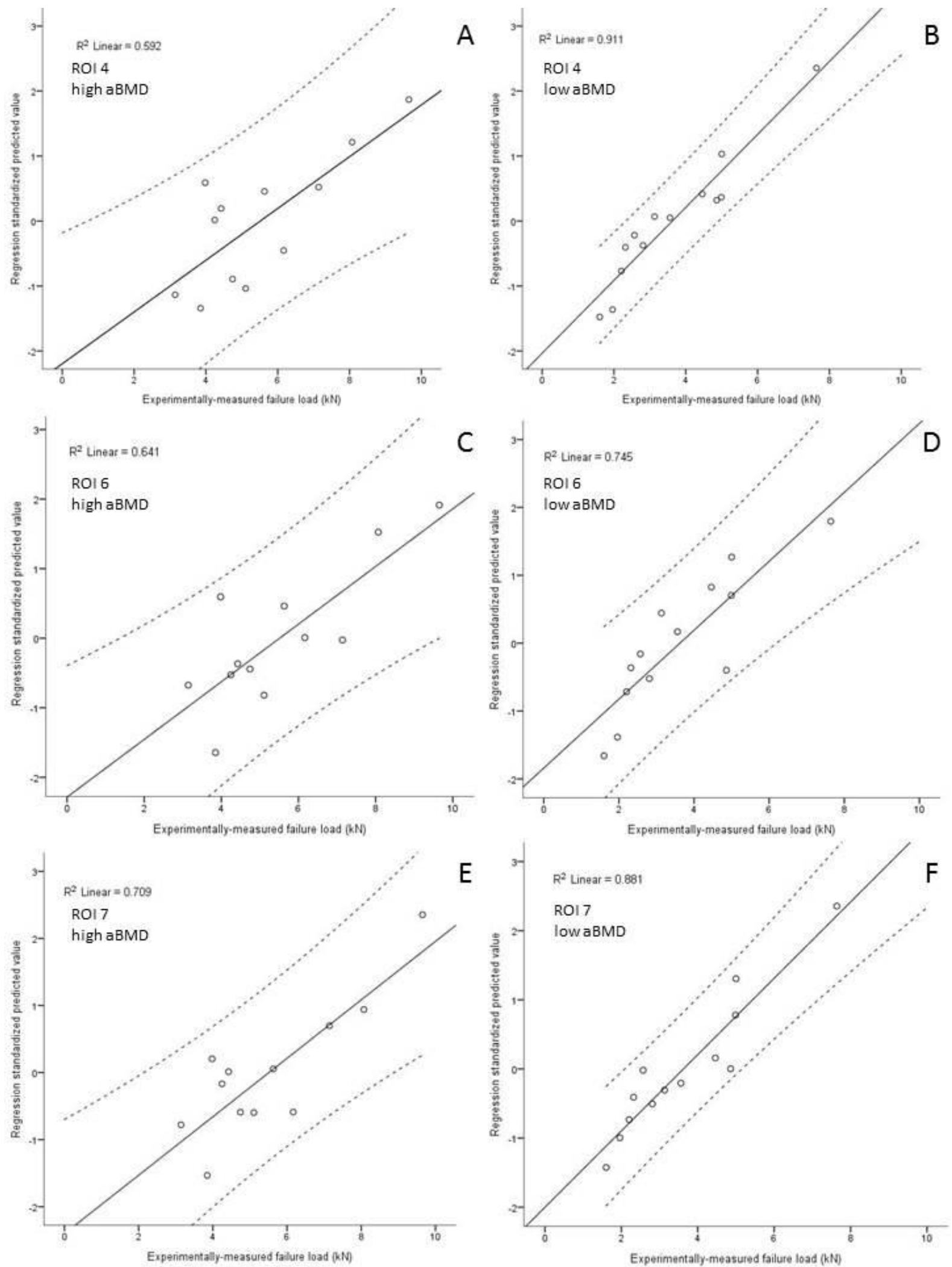